\title{
Correction to: Three Notions of the Global
}

\author{
Sharon Rider
}

\section{Correction to:}

Chapter 4 in: S. Rider et al. (eds.), World Class Universities, Evaluating Education: Normative Systems and Institutional Practices, https://doi.org/10.1007/978-981-15-7598-3_4

The chapter was inadvertently published with one of the references and the citations excluded in the article.

This error has now been corrected by including the citations and the reference. 